\title{
Usefulness of three-dimensional image navigation system for evaluation of hepatic artery before living donor liver transplantation: a case report
}

\author{
Michinori Matsumoto ${ }^{*} \mathbb{D}$, Shigeki Wakiyama, Hiroaki Shiba, Yuichi Ishida, Yoshiaki Kita and Katsuhiko Yanaga
}

\begin{abstract}
Background: The evaluation of the hepatic vascular anatomy in living liver donors is increasingly being performed by three-dimensional (3D) computed tomography (CT) angiography. However, details of hepatic artery anatomy obtained by 3D CT angiography are not always superior to those obtained by angiography. Here, we report a case in which the 3D image navigation system helped to detect segment II, III, and IV arteries (A2, A3, and A4, respectively) that individually originated from the proper hepatic artery (PHA); this could not be detected by 3D CT angiography.

Case presentation: A 46-year-old man with end-stage primary biliary cirrhosis was admitted to our hospital for evaluation as a candidate for living donor liver transplantation. The patient's younger sister, aged 43 years, was the only living donor candidate. The predicted left liver graft volume with the middle hepatic vein was found to be $403 \mathrm{~mL}$ using the region-growing method with 3D CT software. This volume was sufficiently large for the recipient because the standard liver volume of the recipient was $1095 \mathrm{~mL}$. 3D CT angiography was performed twice but could not reveal the anatomical structure of the left and middle hepatic arteries. However, simulation using the region-growing method demonstrated individual branching off of $A 2, A 3$, and $A 4$ from the PHA; conventional angiography demonstrated the same results. Each branch was approximately $1 \mathrm{~mm}$ in diameter, which was too small for secure anastomosis. Therefore, we selected the right liver graft for simplicity. The postoperative course of the donor and recipient was uneventful, and they were discharged on postoperative days 10 and 46, respectively.
\end{abstract}

Conclusions: In conclusion, reconstruction of the hepatic vasculature using the 3D software by region-growing method might be a useful adjunct for surgical planning in the evaluation of the hepatic arteries in living liver donors.

Keywords: Three-dimensional computed tomography, Computed tomographic angiography, Three-dimensional image, Region-growing method

\section{Background}

The preoperative evaluation of donors for living donor liver transplantation (LDLT) aims to select a suitable donor with optimal graft quality and to ensure donor safety. Owing to extensive research on and rapid technical development of computed tomography $(\mathrm{CT})$ scanners and

\footnotetext{
* Correspondence: mmatsumoto4@jikei.ac.jp

Division of Hepato-Biliary-Pancreatic Surgery, Department of Surgery, The Jikei University School of Medicine, 3-25-8 Nishi-shinbashi, Minato-ku, Tokyo 105-8461, Japan
}

Springer Open

(c) The Author(s). 2017 Open Access This article is distributed under the terms of the Creative Commons Attribution 4.0 International License (http://creativecommons.org/licenses/by/4.0/), which permits unrestricted use, distribution, and reproduction in any medium, provided you give appropriate credit to the original author(s) and the source, provide a link to the Creative Commons license, and indicate if changes were made.

three-dimensional (3D) workstations [1], the evaluation of hepatic vascular anatomy in living liver donors is increasingly being performed by 3D CT angiography. However, details of hepatic artery anatomy obtained by the 3D CT angiography are not always superior to those obtained by angiography [2]. On the other hand, 3D image navigation software systems enable not only the calculation of total liver volume and the volume of each vessel's (both the portal and hepatic venous branches) territory but also the visualization of the anatomy of the donor's hepatic vessels 
[3], which facilitates preoperative surgical planning. However, to our knowledge, the usefulness of these 3D image navigation software systems for evaluating the anatomy of the hepatic segmental arteries has not yet been reported.

Here, we describe a case in which the $3 \mathrm{D}$ image navigation system could detect the segment II, III, and IV arteries (A2, A3, and A4) that individually originated from the proper hepatic artery (PHA); this phenomenon could not be detected by 3D CT angiography.

\section{Case presentation}

A 46-year-old Japanese man with end-stage primary biliary cirrhosis was admitted to our hospital for evaluation as a candidate for LDLT. On admission, the model for end-stage liver disease (MELD) score was 20, and the updated Mayo risk score was 11.3. The patient's younger sister, aged 43 years, was the only living donor candidate. Her height and body weight were $153 \mathrm{~cm}$ and $57 \mathrm{~kg}$, respectively.

Multidetector row CT (Siemens Somatom Definition Flash, Siemens Healthcare Japan, Tokyo, Japan) was employed for preoperative dynamic CT. As a contrast material, iopamidol (Iopamiron 370, Bayer, Tokyo,
Japan) with an iodine concentration of $370 \mathrm{mg} \mathrm{I} / \mathrm{mL}$ was intravenously administered (600 $\mathrm{mg} \mathrm{I} / \mathrm{kg}$, maximum dose of $150 \mathrm{~mL}$ ) over $30 \mathrm{~s}$. Images in the arterial phase were obtained by the bolus tracking technique, and the scanning began $15 \mathrm{~s}$ after the trigger threshold, which was set at $80 \mathrm{HU}$ above the aortic baseline CT number, was reached.

Figure 1a shows a simulation of donor hepatectomy, which was performed using the region-growing method with the 3D CT software (organ volume analysis (OVA), Liver Segmentation Software, Hitachi Medico, Tokyo, Japan) [3, 4]. The predicted left liver graft volume with the middle hepatic vein (MHV) was $403 \mathrm{~mL}$, which was sufficiently large for the recipient because a ratio of the graft volume to the standard liver volume of the recipient $(1095 \mathrm{~mL})$ was $\geq 35 \%$. 3D CT angiography was performed twice but could not reveal the anatomical structure of the left and middle hepatic arteries (LHA and MHA) in either case (Fig. 1b). The degree of contrast enhancement was quantified by measuring $\mathrm{HU}$ values by positioning the region of interest at the aorta, right hepatic artery (RHA), umbilical portion of the portal vein, and anatomical structures, such as the LHA or

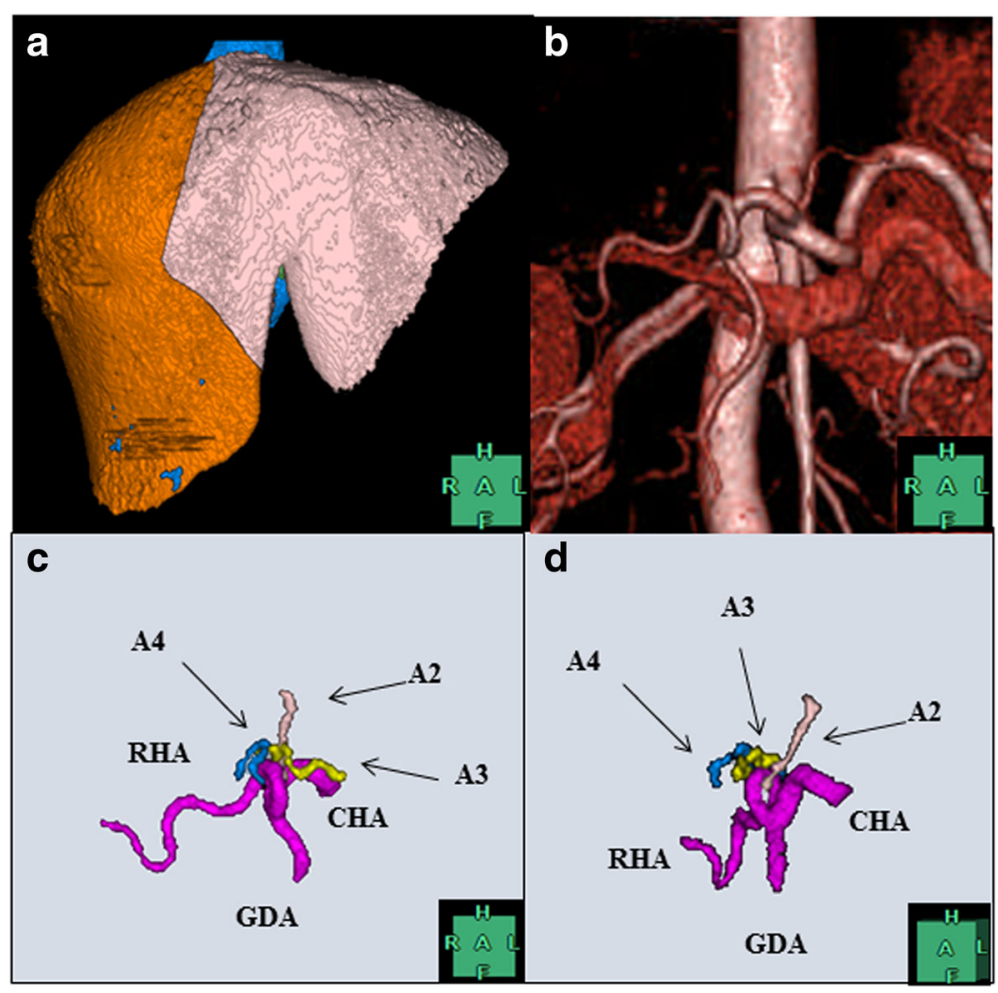

Fig. 1 Donor hepatectomy simulation using the 3D CT software by region-growing method, based on dynamic helical CT of the liver. a The predicted volume of the total liver was $1056 \mathrm{~mL}$, consisting of the right liver (653 mL) and the left liver with the middle hepatic vein (403 mL). b The 3D CT angiography showing no anatomical structure of the LHA and MHA. The simulation using the 3D CT software by region-growing method showing the segment II, III, and IV arteries (A2, A3, and A4 with arrows, respectively) individually originating from the proper hepatic artery. Images of the artery in $\mathbf{c}$ the front view and $\mathbf{d}$ the left anterior oblique view. CHA common hepatic artery, GDA gastroduodenal artery, RHA right hepatic artery 
MHA, which were $363.9 \pm 21.1,304.1 \pm 20.4,123.8 \pm$ 24.0, and $148.8 \pm 26.8 \mathrm{HU}$, respectively. Because CT numbers of anatomical structures, such as the LHA or MHA, were much lower than those of the aorta or the RHA and were comparable to those of the surrounding umbilical portion of the portal vein, the LHA and MHA were deleted in the process of constructing the image of 3D CT angiography. An experienced radiologist and several surgeons suspected the LHA and MHA to have individually originated from the PHA. Subsequently, the hepatic arteries were also extracted using the regiongrowing method similar to the method used for extracting the liver parenchyma, portal vein, and hepatic vein: after setting a seeding point, the region was extended to neighboring voxels if the $\mathrm{CT}$ density of the voxel was within a specified range, which resulted in A2, A3, and A4 individually branching off from the PHA (Fig. 1c, d). To achieve the aforementioned diagnostic goal, conventional angiography was performed, which demonstrated that A2, A3, and A4 individually originated from the PHA, as demonstrated by 3D CT software (Fig. 2). Each branch was approximately $1 \mathrm{~mm}$ in diameter, which was considered as too small for secure anastomosis. If we had selected the left liver as graft with the MHV, we would have had to anastomose up to three branches. Therefore, the donor's right liver was selected. The postoperative course of the donor and recipient was uneventful, and they were discharged on postoperative days 10 and 46 , respectively.

\section{Discussion}

The incidence of three pedicles from the LHA was 3.4\% in the left liver graft [5]. Although successful segmental resection and anastomosis for the three pedicles from the LHA of living donors has been reported [5], we selected the right liver graft for simplicity.

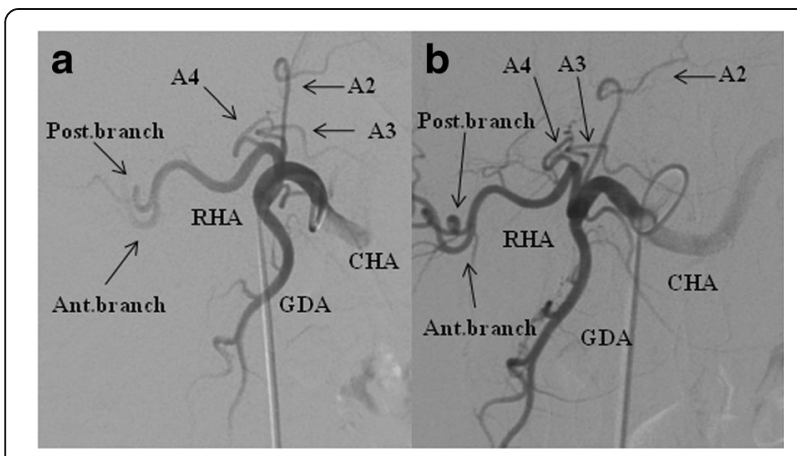

Fig. 2 Angiography showing the segment II, III, and IV (A2, A3, and A4 with arrows, respectively) arteries individually originated from the proper hepatic artery like the simulation. In $\mathbf{a}$ the front view and $\mathbf{b}$ the left anterior oblique view. CHA common hepatic artery, GDA gastroduodenal artery, RHA right hepatic artery, Post. branch posterior branch of RHA, Ant. branch anterior branch of RHA
3D image of A2, A3, and A4 could not be reconstructed for the following reasons: (1) firstly, low enhancement of A2, A3, and A4: one of the most important parameters to minimize partial volume effects in improving spatial resolution in CT angiography is a contrast density of $>250 \mathrm{HU}$, which enables a continuous visualization of the arteries as small as $0.5 \mathrm{~mm}$ in diameter [6]. In the current case, the CT numbers of the aorta and RHA, which could be successfully reconstructed in the 3D image, were $>250 \mathrm{HU}$, whereas those of the anatomical structures, such as the LHA or MHA, were considerably <250 HU. (2) Secondly, slow injection speed of the contrast material: in a comparison of an infusion rate of $2 \mathrm{~mL} / \mathrm{s}$ with $4 \mathrm{~mL} / \mathrm{s}$, the visualization rates of the segmental hepatic arteries improved from $11-34 \%$ at $2 \mathrm{~mL} / \mathrm{s}$ to $76-89 \%$ at $4 \mathrm{~mL} / \mathrm{s}$ [2]. In the current case, the infusion rate was $3.1 \mathrm{~mL} / \mathrm{s}$, and $\mathrm{A} 2, \mathrm{~A} 3$, and $\mathrm{A} 4$ were not regarded as extrahepatic but as segmental arterial branches. These segmental branches were deleted in the process of constructing the 3D CT angiography image, even when the infusion rate was $4 \mathrm{~mL} / \mathrm{s}$, because of the poor continuous arterial enhancement similar to that in a previous study [2]. Other less invasive modalities are warranted to comprehend the hepatic arterial anatomy.

The advantages of OVA using the region-growing method with 3D CT software are as follows: (1) 3D imaging of liver structures, (2) detailed volumetric analyses based on portal perfusion, and (3) quantitative estimates of the venous drainage area, enabling the investigation of unknown fields that cannot be examined using conventional two-dimensional modalities such as handtracing volumetry $[3,4]$. According to the manufacturer's information for OVA, the software is based on an algorithm that defines the perfusion area of each portal branch based on the direction and diameter of vessels $[3,4]$. In the present case, the region-growing method was applied to the 3D image reconstruction of the hepatic arteries. Compared with the extraction of the arteries using semi-automatic tracing of the arteries, that using the region-growing method produced a more objective 3D image because extracted arterial images were reconstructed based on the $\mathrm{CT}$ densities of the voxels within the specified range of the hepatic arteries and not of the surrounding portal vein. The reconstruction of 3D image of these small less-contrasted branches using the region-growing method was visually successful.

\section{Conclusions}

In conclusion, the reconstruction of hepatic vasculature using the region-growing method with $3 \mathrm{D}$ CT software might be a useful adjunct for surgical planning in the evaluation of the hepatic arteries in live liver donors. 


\section{Abbreviations}

3D: Three-dimensional; CT: Computed tomography; HU: Hounsfield unit;

LDLT: Living donor liver transplantation; LHA: Left hepatic artery;

MELD: Model for end-stage liver disease; MHA: Middle hepatic artery;

MHV: Middle hepatic vein; PHA: Proper hepatic artery; RHA: Right hepatic artery

\section{Funding}

The authors declare no financial or any other type of support.

\section{Authors' contributions}

All authors participated in the management of the patient in this case report. MM drafted the manuscript. SY is one of the chief surgeons of our hospital who supervised the cases and also supervised the writing of the manuscript. KY is a chairperson of our department who supervised the entire process. All authors read and approved the final manuscript.

\section{Consent for publication}

The patient has provided permission to publish these features of the cases, and the identity of the patient has been protected.

\section{Competing interests}

The authors declare that they have no competing interests.

\section{Publisher's Note}

Springer Nature remains neutral with regard to jurisdictional claims in published maps and institutional affiliations.

Received: 6 April 2017 Accepted: 11 July 2017

Published online: 28 July 2017

\section{References}

1. Kamel IR, Kruskal JB, Pomfret EA, Keogan MT, Warmbrand G, Raptopoulos V. Impact of multidetector $C T$ on donor selection and surgical planning before living adult right lobe liver transplantation. Am J Roentgenol. 2001;176:193200.

2. Sakai H, Okuda K, Yasunaga M, Kinoshita HR, Aoyagi S. Reliability of hepatic artery configuration in 3D CT angiography compared with conventional angiography_-special reference to living-related liver transplant donors. Transpl Int. 2005;18:499-505.

3. Saito S, Yamanaka J, Miura K, Nakao N, Nagao T, Sugimoto T, et al. A novel 3D hepatectomy simulation based on liver circulation: application to liver resection and transplantation. Hepatology. 2005;41:1297-304.

4. Mise Y, Tani K, Aoki T, Sakamoto Y, Hasegawa K, Sugawara Y, et al. Virtual liver resection: computer-assisted operation planning using a threedimensional liver representation. J Hepatobiliary Pancreat Sci. 2013;20:157-64.

5. Takatsuki M, Chiang YC, Lin TS, Wang CC, Concejero A, Lin CC, et al. Anatomical and technical aspects of hepatic artery reconstruction in living donor liver transplantation. Surgery. 2006;140:824-8. discussion 9.

6. Luboldt W, Weber R, Seemann M, Desantis M, Reiser M. Influence of helical $\mathrm{CT}$ parameters on spatial resolution in $\mathrm{CT}$ angiography performed with a subsecond scanner. Invest Radio. 1999;34:421-6.

\section{Submit your manuscript to a SpringerOpen ${ }^{\circ}$ journal and benefit from:}

- Convenient online submission

- Rigorous peer review

- Open access: articles freely available online

- High visibility within the field

- Retaining the copyright to your article 